\title{
An Accuracy Assessment of Cartesian-Mesh Approaches for the Euler Equations
}

\author{
William J. Coirier* \\ NASA Lewis Research Center \\ Cleveland, Ohio USA \\ and \\ Kenneth G. PowelI** \\ The University of Michigan \\ Ann Arbor, Michigan USA
}

\begin{abstract}
A critical assessment of the accuracy of Cartesian-mesh approaches for solving the Euler equations is made. An exact solution of the Euler equations (Ringleb's flow) is used not only to infer the order of error of the Cartesianmesh approaches, but also to compare the magnitude of the error directly to that obtained with a structured mesh approach. The effect of cell merging is investigated as well as the use of two different $K$-exact reconstruction procedures. The solution methodology of the schemes is explained and tabulated results are presented to compare the solution accuracies. Adaptive and uniform mesh refinement is evaluated for Ringleb's flow and the supersonic flow through an axi-symmetric inlet.
\end{abstract}

* Member AIAA. Aerospace Engineer, Doctoral Candidate, The University of Michigan, Department of Aerospace Engineering

** Senior Member AIAA. Associate Professor, Department of Aerospace Engineering

Copyright 1993 by the American Institute of Aeronautics and Astronautics, Inc. No copyright is asserted in the United States under Title 17. U.S. Code. The U.S. Government has a royalty-free license to exercise all rights under the copyright claimed herein for Govemmental purposes. All other rights are reserved by the copyright owner.

\section{Introduction}

With the advent of unstructured meshes, it is becoming possible to perform high quality calculations of flows of increasing geometric and physical complexity. This geometric flexibility is obtained by using an unstructured grid data structure that, if formulated properly, can allow mesh enrichment by cell division. In this way, unstructured solvers with adaptive mesh refinement can resolve disparate length scales on geometrically complicated domains and perhaps provide a means to achieve automatic mesh convergence. Mesh redistribution schemes have the benefit of being able to use existing, structured mesh flow solvers with few modifications, but suffer from the constraints borne by the structured mesh data structure. As pointed out in [1], adaptive mesh refinement via mesh or cell enrichment is superior to mesh redistribution for precisely this reason, although both schemes can be an improvement to the non-adaptive approach. Through the proper formulation of data structures and by an efficient implementation of non-traditional algorithms, unstructured meshes approaches can compete with and complement standard, structured-mesh approaches.

The method assessed here is a Cartesian-mesh approach. Cartesian-mesh approaches have been in the literature for a number of years. In [2] and [4] unsteady shock hydrodynamic problems were computed on a Cartesian mesh on a Cartesian domain. Adaptive mesh refinement was achieved by adding collections of cells, grouped into contiguous grids about fronts in the field, using front detection algorithms 
based upon pattern recognition. In [5], a similar approach was used to compute highly resolved, unsteady shock hydrodynamic problems, where the grids used were based upon a background, body fitted mesh. By using unique data structures coupled with non-traditional algorithms, these approaches were able to compute highly resolved flows with multiple fronts and length scales.

Cartesian-mesh based approaches have been used with great success for computing flows about complicated geometries by solving the full potential equation in a finite-element formulation in [6]. In this application, full use was made of the underlying hierarchical structure of the grid through the use of an octree based data structure. The Cartesian-mesh approach was extended to solving the Euler equations in a finite volume formulation in [7] for computing the transonic flow about multi-element airfoils, although no mesh refinement was performed. The basic premise behind the Cartesian-mesh approach is the use of a regular, background mesh composed entirely of Cartesian cells to allow a local description of arbitrarily complex boundaries. This results in either irregularly shaped cells at the boundaries (for a finite volume approach) or special boundary procedures (for a finite difference approach). For the finite-volume formulation, it is preferred to "cut" the geometry out of the background mesh, resulting in irregularly shaped cells upon which a flux balance must be performed. It is the treatment of the boundaries and the resulting irregularly shaped cells that has been a pacing item for the finite volume form of this technique. In [8], a unique method of cell cutting for the finite volume variant was shown and impressively demonstrated for an unsteady, geometrically complicated flow.

In $[3,8,9,10,11,12,13]$ reliance upon a locally structured background mesh was used for gradient reconstruction and to help formulate the flux balance on irregular boundaries and coarse/fine mesh interfaces. This close coupling to the background structured mesh is advantageous when refinement is made by adding grid patches, but truly local refinement could result in patches of only single cells. In [14] a linear reconstruction technique (Green-Gauss reconstruction) was used on the Cartesian-cell mesh. This reconstruction technique is locally second order accurate, and is used in many unstructured mesh approaches. This decoupling of the reconstruction process from the flux computation sets this approach apart from the previous Cartesiancell work in that a true decoupling from the background mesh was achieved, resulting in what at first appears to be an unstructured mesh approach. But, by exploiting the hierarchical properties obtainable through the genesis of the mesh, the resulting approach can be more aptly named a hierarchically-structured, Cartesian-mesh approach. This approach was applied successfully to investigate many interesting flow fields $[15,18,21]$, but the accuracy of the approach has not been benchmarked. Since this approach would like to promise automatic mesh generation along with automatic mesh convergence, the accuracy of the approach needs to be closely examined.

This study performs a critical assessment of a Cartesianmesh approach by using an analytical solution to the Euler equations (Ringleb's flow) and directly compares the solution accuracy to that obtained with a (streamline-aligned) structured mesh calculation. The effect of adaptive mesh refinement is evaluated using Ringleb's flow, which is smooth and analytic, and the non-smooth supersonic flow through an axi-symmetric, mixed compression inlet. The framework of using an exact, analytic solution to the Euler equations allows other important procedures to be quantitatively analyzed. Two linear reconstruction procedures (Green-Gauss and Minimum-Energy) are evaluated and the approach of cut-cell data merging is outlined and examined in the framework of Ringleb's flow.

\section{A Cartesian-Mesh Approach}

The approach presented here solves the Euler equations of compressible fluid dynamics using a cell centered, finite volume, upwind scheme. A linear reconstruction of the primitive variables is used to determine the state quantities at cell to cell interfaces which are then used to compute the flux using an approximate Riemann solver. Typically a coarse, base mesh is generated, upon which a solution is obtained. Then, based upon this (coarse) solution, the mesh is adaptively refined, and a new solution is found on this refined mesh. This process is repeated a number of times, solving and then refining, until a specified degree of adaptation is achieved. This automatic, adaptive mesh refinement, coupled with the automatic mesh generation, attempts to gain grid converged solutions with minimal user intervention. The following sections briefly outline the approach. 


\section{II.a Mesh Generation/Data Structure}

One objective of this approach is to create a mesh generation procedure that can be automated for complex geometries. In addition, the resulting method and data structure should easily allow for mesh refinement and mesh coarsening. The approach presented here is based upon using Cartesian cells of unit aspect ratio that are "cut" into irregular cells whenever the boundary of a body intersects any of its surfaces. By using Cartesian cells of unit aspect ratio, and by splitting these cells into 4 children the resulting mesh is highly uniform and smooth in uncut regions. The mesh generation proceeds automatically, once the geometry is suitably defined, by finding the intersections of the body surfaces with each cell boundary, and determiningwhether these intersections (if any) satisfy a simple set of cell cutting rules. If a cell is intersected, and it doesn't satisfy the cell cutting rules, it is refined (split into 4 cells). This proceeds recursively over all current cells in the mesh, only refining the offending cells, until the resulting mesh is suitable for the cutting of the geometry. Arbitrary numbers of bodies can be used to form the geometry, where each body can be represented by discrete (pointwise data) or arbitrary functional descriptions. The staggered biplane configuration computed in [7] is used here to illustrate the mesh generation process. Figures 1 and 2 show a portion of the domain before and after cutting the geometry out of the background mesh.

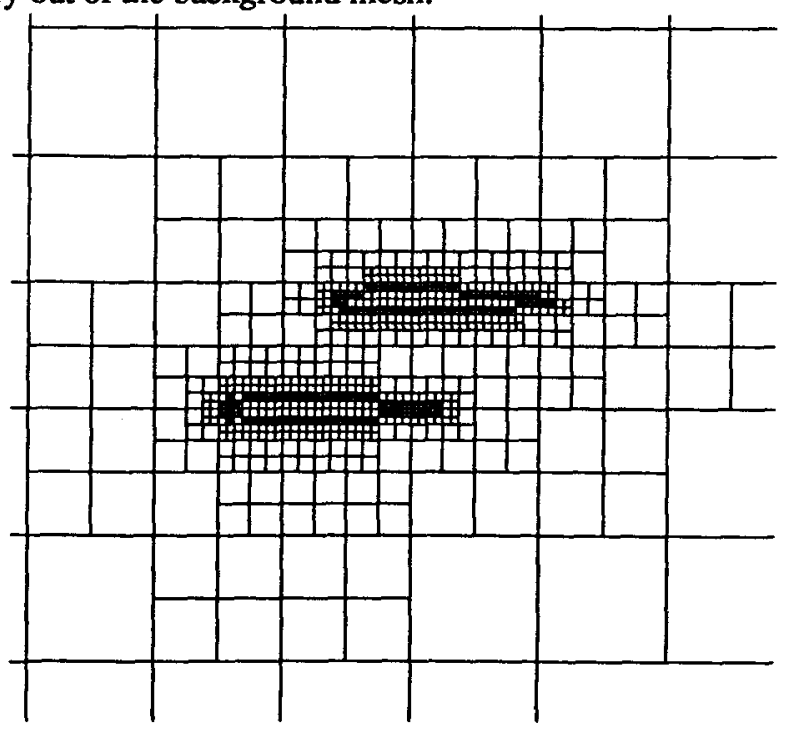

FIGURE 1. Staggered Biplane mesh: Prior to Cell Cutting.

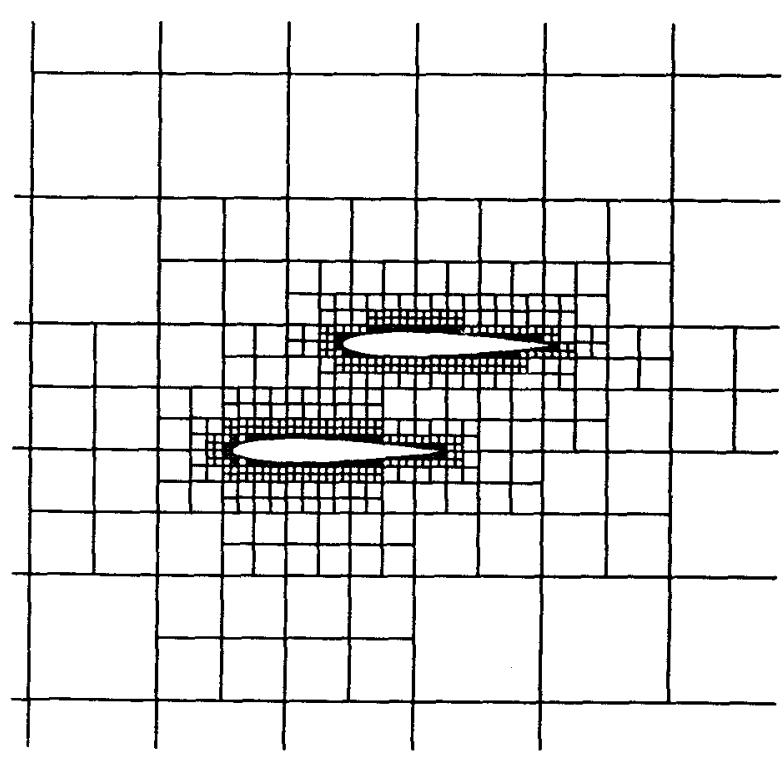

FIGURE 2. Staggered Biplane mesh: Immediately After Cell Cutting.

As opposed to using an array or some other approach using "flat-lists", a hierarchical data structure is used to store the mesh. The data structure is based upon a binary tree, where each node of the tree represents a cell at some stage of the cell spawning process. Figure 3 is an illustration showing the stages of splitting a Cartesian cell isotropically into four children. Referring to this figure, consider cell $A$, which is first split in $x$. This results in 2 cells, B and $C$, that are the children of $A$. If the se are then split in $y$, the result is a set of 4 children, cells D, E, F and G, that are contained geometrically within the parent cell, $\mathrm{A}$, and hierarchically below $A$ in the tree.

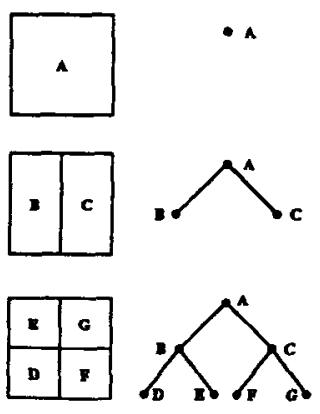

FIGURE 3. Illustration of Cell Splitting/Tree Structure 
The data structure mimics this hierarchical nature by having a pointer to the cell parent and two pointers to its children, in addition to geometric data (cell centroid and cell size) and tree level data. It should be noted that if the mesh were everywhere Cartesian, all geometric data could be directly inferred from the tree. Another useful property of the tree data structure is that cell-to-cell connectivity can be inferred from the tree via logical tree traversals based on centroid compares and face-matching procedures. The resulting computer code is written in ANSI standard C to take advantage of dynamic memory allocation, deallocation and self-referential data structures.

\section{II.b Flow Solver Formulation}

The Euler equations are solved using a cell centered, finite volume, upwind based scheme. Roe's approximate Riemann solver [20] is used to formulate the equivalent onedimensional flux through the cell-to-cell interfaces. The states at these interfaces are found using a two-dimensional interpolation of cell primitives that is formed using linear reconstruction. Two reconstruction procedures are explained and investigated here: Green-Gauss and $\mathrm{L}_{2}$ (also known as Minimum-Energy). The resulting schemes, when applied in one-dimension, are similar to Fromm's scheme. When a limiting procedure is used, the reconstructed states are limited using a minimum-modulus type limiter. A generic multi-stage scheme is used to drive the residuals to zero. The solution methodology follows the standard finite-volume approach, in that the procedure can be broken into 3 separate stages: reconstruction, flux computation and evolution. A brief outline of each segment is given below.

\section{Reconstruction}

The primitive variables are reconstructed in each cell using a linear reconstruction. The process of reconstruction to arbitrary degrees of accuracy on unstructured meshes is presented in detail by Barth in $[16,17]$ and by Godfrey et.al. in [19], of which a distilled presentation of the $L_{2}$ reconstruction is shown below. The process of reconstruction can be considered as the discrete inverse of a cell averaging process. In other words, given the distribution of a function within a cell, one can compute the cell average by

$$
\bar{v}=\frac{1}{\mathrm{~A}} \int_{\mathrm{A}} \mathrm{u}(\mathrm{x}, \mathrm{y}) \mathrm{d} A
$$

Reconstruction solves the inverse of this problem: find the expansion about the cell centroid to $k$-th order, $u^{k}(x, y)$, using the cell averaged data of the cell to be reconstructed and a set of support cells. The support of cells used in the reconstruction is typically taken to be nearest neighbor cells; that is cells that share a face and/or a vertex with the cell where the reconstruction is desired.

\section{Minimum-Energy Reconstruction}

By expanding $u^{k}(x, y)$ in terms of zero mean basis polynomials, conservation of the mean of the object cell is ensured, resulting in the general expansion

$$
u^{k}(x, y)=\bar{u}+\sum_{j} \alpha_{j} \psi_{j}(x, y)
$$

where the $\psi_{j}$ are constructed such that their cell average is identically zero. The reconstruction process finds the $\alpha$. above by minimizing the least square error, $S$, with respect to the neighbor cell averages of the $u^{k}$. That is, minimize with respect to the $\alpha_{j}$

$$
s=\sum_{n} \omega_{n}\left(u^{k}\left(\bar{x}_{n}, \bar{y}_{n}\right)-\bar{u}_{n}\right)^{2}
$$

This results in a linear system for the $\alpha_{j}$

$$
L_{i j} \alpha_{j}=b_{i}
$$

where

$$
\begin{aligned}
L_{i j} & =\sum_{n} \omega_{n} \psi_{i}\left(\bar{x}_{n}, \bar{y}_{n}\right) \psi_{j}\left(\bar{x}_{n}, \bar{y}_{n}\right) \\
b_{i} & =\sum_{n} \omega_{n}\left(\bar{u}_{n}-\bar{u}_{)} \Psi_{i}\left(\bar{x}_{n}, \bar{y}_{n}\right)\right.
\end{aligned}
$$

For a given mesh, the $\mathrm{L}_{\mathrm{ij}}$ is only dependent upon the geometry, so it can be inverted beforehand. This preprocessing of the reconstruction makes it efficient in that only a simple sum is needed to compute the reconstructed solution. For the work considered here, the expansion is taken 
out only to $k=1$, resulting in a linear expansion, with the meaningful expression for the reconstruction

$$
u^{1}=\bar{u}+u_{x}(x-\bar{x})+u_{y}(y-\bar{y})
$$

It should be pointed out that this reconstruction needs no special ordering of points, requires a minimum of two neighbors and is obtained by a simple summation over the support cells. This results in a second-order accurate reconstruction of the local solution in a cell and reconstructs linear functions exactly. Unless noted otherwise, all calculations were computed using this reconstruction procedure.

\section{Green-Gauss Reconstruction}

Green-Gauss reconstruction is another type of linear reconstruction that is commonly used in unstructured grid solvers. Green's theorem applied to a scalar function relates the volumetric integral of the gradient of the function to its surface integral over the surface of the bounding volume. If the gradient is assumed to be constant over the cell the reconstructed gradient can be found as

$$
\nabla \mathrm{u}=\frac{1}{\mathrm{~A}} \oint_{\Gamma} \mathrm{u} \hat{\mathrm{n}} d \Gamma
$$

The line integral in (8) is ordered counter-clockwise, which requires the neighbor cell averaged data also to be ordered counter-clockwise. The line integral is computed using second-order Gaussian quadrature which results in a summation o rer the (ordered) neighbor cells. The reconstruction ob 'ained is second order accurate, reconstructs linear functions exactly, and has a marginally smaller truncation error than the Minimum-Energy reconstruction on strictly uniform Cartesian meshes.

\section{Flux Computation}

The Euler equations are solved using a standard, cell centered, finite-volume scheme. After converting the divergence of the fluxes into a surface integral, the time rate of change of the cell averages of the conserved variables is

$$
\frac{\partial \overline{\mathrm{q}}}{\partial \mathrm{t}}=-\frac{1}{\mathrm{~A}} \oint_{S} \mathrm{~F} \bullet \hat{\mathrm{nd}} \mathrm{S}
$$

This surface integral is approximated using 2nd-order Gaussian quadrature. It is pointed out in [17] that it is necessary to use a quadrature of at least the same order as the reconstruction order, and that the accuracy achieved using a quadrature of higher order does not warrant the extra effort. Second order Gaussian quadrature results in evaluating the kernel of (9) at the midpoints of all the edges representing the mesh. Replacing the right hand side of (9) with the numerical quadrature, the semi-discrete form of the equations is reduced to

$$
\frac{\partial \bar{q}}{\partial \mathrm{t}}=-\frac{1}{\mathrm{~A}} \sum \Phi\left(\mathrm{U}_{\mathrm{L}}, \mathrm{U}_{\mathrm{R}}\right) \Delta \mathrm{S}
$$

The sum is taken over all faces of the cell, and the left and right states of the Riemann solver are found using the reconstructed solutions in each cell evaluated at the Gauss points. The numerical flux, $\Phi$, is formed in the face normal coordinate system in a standard upwind formulation, where the numerical flux difference is formed using Roe's linearized Riemann solver.

\section{Evolution}

Since obtaining steady-state solutions is the goal, a spatially varying time step is used by advancing all cells in the mesh at a constant Courant number. This is quite necessary since cell cutting and mesh adaptation can result in widely varying cell areas across the mesh. A generic 3 stage explicit scheme is used to drive down the residuals, with stage coefficients of $(0.18,0.5,1.0)$ and a Courant number of 1.3 .

\section{II.c Mesh Adaptation}

Adaptive mesh refinement is achieved using the refinement criteria presented in [21] and [15]. Briefly, refinement is based upon a statistical description of two refinement parameters that characterize the local compressibility and rotationality of the fluid. These parameters are

$$
\begin{aligned}
& \tau_{\mathrm{r}}=|\nabla \times \mathrm{u}| \mathrm{1}^{\frac{3}{2}} \\
& \tau_{\mathrm{c}}=|\nabla \bullet \mathrm{u}| 1^{\frac{3}{2}}
\end{aligned}
$$


where the characteristic cell length is taken to be $1=\sqrt{\mathrm{A}}$. Cells are refined that have cell lengths greater than some user specified value and either refinement parameter is greater than its standard deviation from zero. Cells are a candidate for coarsening if both parameiers are less than some fraction of the standard deviation from zero (taken here to be one tenth).

The following figures illustrate the Cartesian-mesh approach for the staggered biplane configuration computed in [7]. The two elements are NACA 0012 airfoils, staggered $1 / 2$ chord length in the chordwise and pitch directions. The free stream Mach number is $M_{\infty}=0.7$ and the configuration is at $\alpha=0$ degrees. The computation is made through 4 levels of refinement beyond the base mesh. Figures 4 and 5 show a portion of the adapted flow field and mesh.

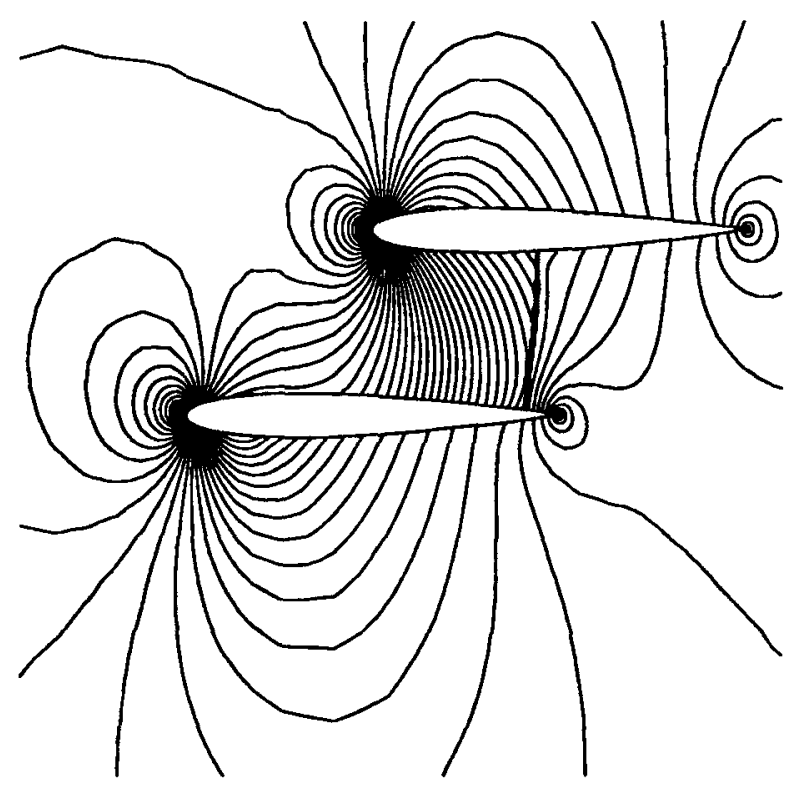

FIGURE 4. Pressure Contours: Staggered Biplane Configuration

All structured and Cartesian calculations reported in this study were converged to a drop in the $L_{2}$ norm of the residual of the continuity equation of at least 6 orders of magnitude. All of the calculations were performed on IBM RS6000 workstations. The aggregate processing rates for the Cartesian-mesh calculations on a Model 560 were approximately $200 \mu$ seconds/cell/iteration for the (un-limited) Ringleb's flow calculations and approximately $300 \mu$ seconds/cell/iteration for the (limited) supersonic inlet calculations (shown in a later section).

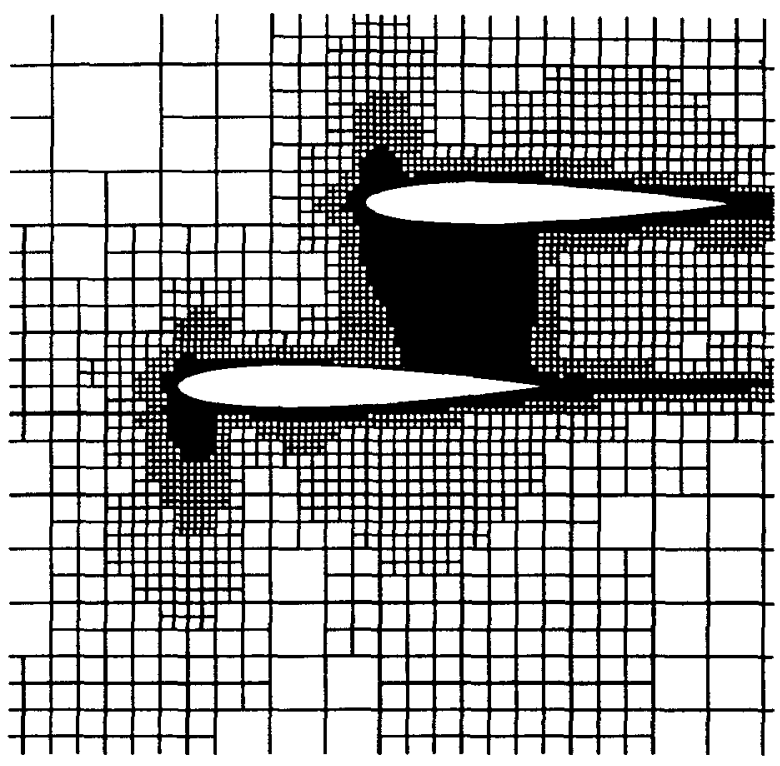

FIGURE 5. Adapted mesh: Staggered Biplane Configuration

\section{Accuracy Assessment: Ringleb's Flow}

Ringleb's flow is a hodograph solution to the Euler equations [22], and has been used to assess the accuracy of other structured-and unstructured-mesh approaches $[17,19,23]$. A variety of flows can be attained, depending upon the choice of parameters used. The solution is parameterized in terms of the total velocity, q, and streamline constant, $\mathrm{k}$ as

$$
\begin{gathered}
x(q, k)=\frac{1}{2 \rho}\left(\frac{2}{k^{2}}-\frac{1}{q^{2}}\right)-\frac{J}{2} \\
y(q, k)= \pm \frac{1}{k \rho q} \sqrt{1-\left(\frac{q}{k}\right)^{2}} \\
c=\sqrt{1-\frac{(\gamma-1)}{2} q^{2}} \\
\rho=c^{\frac{2}{\gamma-1}}
\end{gathered}
$$




$$
J=\frac{1}{c}+\frac{1}{3 c^{3}}+\frac{1}{5 c^{5}}-\frac{1}{2} \log \left(\frac{1+c}{1-c}\right)
$$

where the density, $\rho$, is made non-dimensional by its stagnation value and all speeds are made non-dimensional by the stagnation sound speed. The flow angle $\theta$ is related to the streamline constant and total velocity by

$$
\theta=2 \pi-\operatorname{asin}\left(\frac{q}{k}\right)
$$

The flow in the first quadrant is computed that is bounded by the streamlines $\mathrm{k}=0.75$ and $\mathrm{k}=1.5$. The outflow boundary is situated along the $y=0$ line of symmetry and the inflow boundary is along the isovelocity line of $q=0.5$. The resulting flow has a subsonic inflow and a mixed supersonic/subsonic outflow. Figure 6 shows contours of the Mach number of the flow field obtained with these parameters. As can be seen from the figure, the flow can be visualized as a transonic, accelerating flow contained between two curved streamlines.

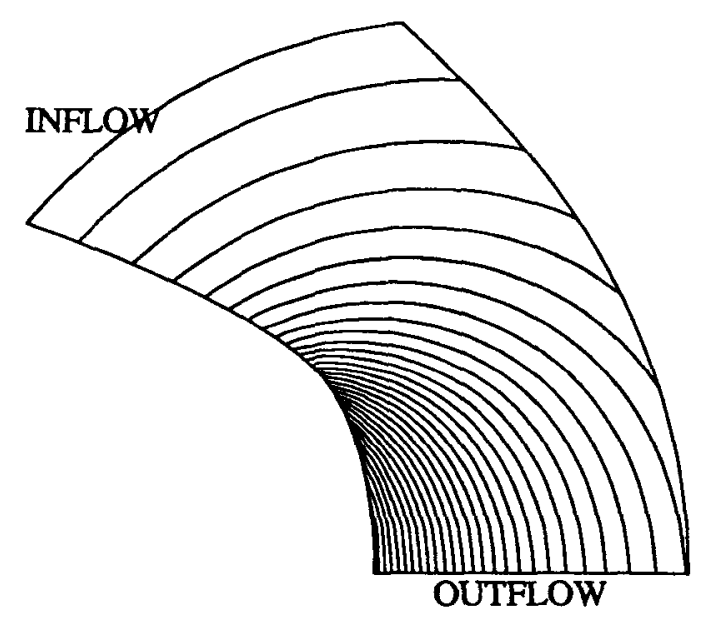
FIGURE 6. Mach Number Contours of Ringleb's
Flow

If one defines the error for the $i$-th cell as

$$
e_{i}=\left|\bar{\rho}_{i}-\rho_{\text {exact }}\left(\bar{x}_{i}, \bar{y}_{i}\right)\right|
$$

then the $L_{p}$ norms of the error are

$$
\mathrm{L}_{\mathrm{p}}=\left(\frac{\sum e_{i}^{P}}{N}\right)^{\frac{1}{P}}
$$

First, an assessment of the order of accuracy and the magnitude of the error using the Cartesian-mesh approach is made. The order is inferred by evaluating the behavior of the error norms with increasing mesh refinement, while the magnitude of the error is assessed by comparing the error norms directly with those obtained from a structured mesh solver. This accuracy assessment provides a framework to quantitatively analyze other relevant procedures. The accuracy of the Green-Gauss and Minimum-Energy are compared to each other, and the process of cut cell data merging is evaluated.

The structured-grid flow solver uses Fromm's differencing of the primitive variables on a coordinate-by-coordinate basis. Ro's linearized Riemann solver is used to compute the fluxes through the cell interfaces. Care is taken in the formulation of the boundary procedures so that a direct comparison of the two codes yields meaningful results. Slip boundary conditions are applied by extrapolating the pressure to the Gauss points in a manner consistent with the interior scheme. At the subsonic inflow, a boundary procedure based upon constant total conditions and an extrapolated Riemann invariant (as in [24]) is used. Roe's approximate Riemann solver is used at the mixed supersonic/subsonic outflow boundary The left and right states are supplied to the flux function from extrapolated and exact conditions evaluated at the Gauss points.

\section{III.a Structured Grid Results}

The meshes used for the structured grid calculations have a family of coordinate lines lying along the exact solution streamlines. The other coordinate line family was generated using a sinusoidal blending of the streamline and isovelocity constants. A sample structured mesh is shown in Figure 7 with 400 cells. A sequence of successively finer meshes of $10 \times 10,20 \times 20,40 \times 40$ and $80 \times 80$ cells were used to compute Ringleb's flow, upon which the solution error norms were computed. The norms are tabulated in Table $I$. 
TABLE I. Structured Grid Error Norms

\begin{tabular}{|l|l|l|l|}
$\mathbf{N}$ & $L_{1}$ & $L_{2}$ & $L_{\infty}$ \\
\hline 100 & $2.368 \mathrm{e}-03$ & $2.796 \mathrm{e}-03$ & $1.066 \mathrm{e}-02$ \\
\hline 400 & $4.517 \mathrm{e}-04$ & $5.352 \mathrm{e}-04$ & $2.700 \mathrm{e}-03$ \\
\hline 1600 & $1.157 \mathrm{e}-04$ & $1.337 \mathrm{e}-04$ & $6.918 \mathrm{e}-04$ \\
\hline 6400 & $3.050 \mathrm{e}-05$ & $3.514 \mathrm{e}-05$ & $1.745 \mathrm{e}-04$ \\
\hline
\end{tabular}

By plotting the logarithm of the norms against the logarithm of the characteristic cell size, $1 / \sqrt{N}$, one can infer the order of the truncation error form the slope of the plot. A least-squares curve fit of the data gives slopes of the $\mathrm{L}_{1}, \mathrm{~L}_{2}$ and $\mathrm{L}_{\infty}$ norms of 2.08, 2.09 and 1.97 respectively, indicating that the structured scheme is uniformly 2nd order accurate. It should be noted that the mesh used here is quite beneficial: with a closer examination, one can see that by virtue of the mesh generation, not only is one family of mesh lines aligned exactly with the flow streamlines, but clustering is achieved near the place of minimum radius of curvature of the left wall. Indeed, in a later section, it is shown that the adaptively refined mesh is clustered in this region.

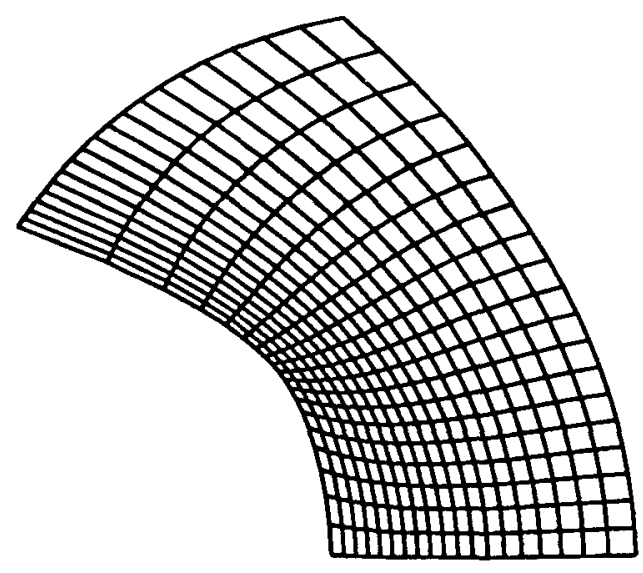

FIGURE 7. Structured mesh: 400 Cells

\section{III.b Uniformly Refined Cartesian Results}

Next, the Cartesian-mesh approach is used to compute Ringleb's flow on a sequence of successively finer uni- form Cartesian meshes. The uniform meshes are generated by recursively refining a set number of levels below the root of the tree, and then cutting the geometry out of the mesh. The number of levels below the root cell characterizes the fineness of the uniform meshes, which we will refer to as the mesh base level, $\mathrm{L}_{0}$.

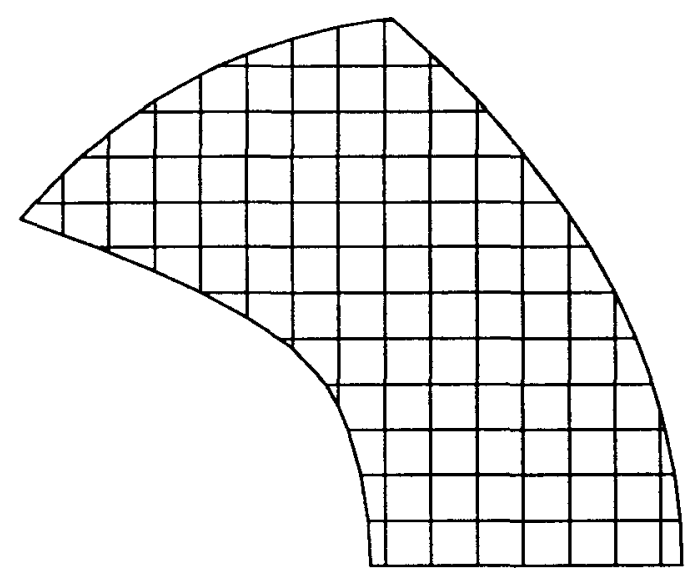

FIGURE 8. Uniform Cartesian Mesh, $L_{0}=4$.

Figure 8 shows the coarsest mesh, which is 4 levels below the root cell, hence at a mesh base level of $L_{0}=4$. Uniformly refined calculations were made for base grid levels of 4,5,6 and 7, of which the error norms are tabulated in Table II.

TABLE II. Uniformly Refined Cartesian Error Norms

\begin{tabular}{|l|l|l|l|}
$\mathrm{N}$ & $L_{1}$ & $L_{2}$ & $L_{\infty}$ \\
\hline 118 & $5.345 \mathrm{e}-03$ & $8.658 \mathrm{e}-03$ & $4.497 \mathrm{e}-02$ \\
\hline 417 & $1.571 \mathrm{e}-03$ & $2.554 \mathrm{e}-03$ & $1.458 \mathrm{e}-02$ \\
\hline 1578 & $4.236 \mathrm{e}-04$ & $7.394 \mathrm{e}-04$ & $6.928 \mathrm{e}-03$ \\
\hline 6134 & $9.793 \mathrm{e}-05$ & $1.983 \mathrm{e}-04$ & $2.674 \mathrm{e}-03$ \\
\hline
\end{tabular}

A least-squares curve fit of the uniformly refined norm data yields slopes for the $\mathrm{L}_{1}, \mathrm{~L}_{2}$ and $\mathrm{L}_{\infty}$ norms of 2.02 , 1.91 and 1.40 , respectively. Using the two finest meshes one obtains slopes for the $\mathrm{L}_{1}, \mathrm{~L}_{2}$ and $\mathrm{L}_{\infty}$ norms of 2.16, 1.94 and 1.40, respectively. These slopes indicate that the Cartesian-cell based scheme is globally 2 nd order accurate and that the local error is greater than 1st order accurate but not quite 2 nd order. An analysis of the effect of the boundary cells upon the solution accuracy is estimated by 
computing error norms separately for the boundary cells, and then computing the slopes as above. The computed slopes of the boundary cell $\mathrm{L}_{1}, \mathrm{~L}_{2}$ and $\mathrm{L}_{\infty}$ norms were 1.68, 1.49 and 1.40. Although the local error is degraded by the irregularity in the mesh due to the cut cells/boundaries, the scheme remains globally second order accurate.

\section{III.c Adaptively Refined Cartesian Results}

The effect of adaptive refinement is assessed next. Beginning at a base uniform mesh of level $\mathrm{L}_{0}=4$, adaptation proceeds through 4 levels of refinement. The refinement is made according to the rotationality and compressibility parameters described above, although for this irrotational flow, the rotationality parameter is nearly zero and does not effect the refinement topology. Figure 9 shows the adapted mesh that corresponds to a mesh refinement of 2 levels below the base, uniform mesh.

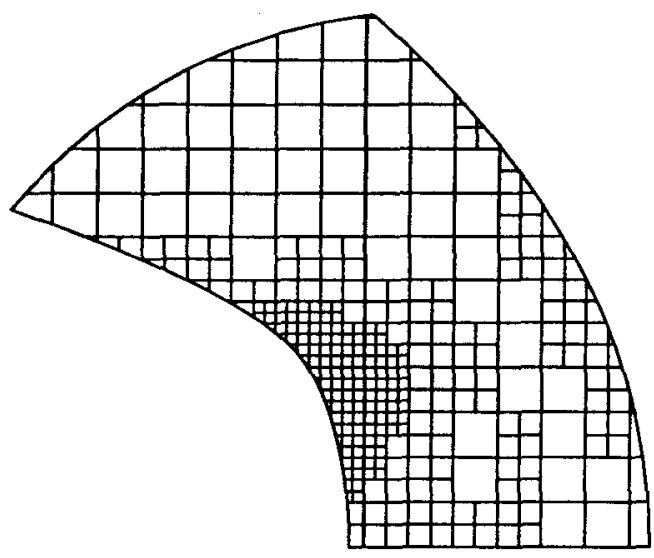

FIGURE 9. Adapted Cartesian Mesh

The adaptively refined norms are tabulated in Table III. The error norms are compared with the characteristic cell size in figures 10,11 and 12 for the structured, uniformly and adaptively refined Cartesian calculations. As is shown in Figures 10 through 12, the $L_{1}$ and $L_{2}$ norms continue to behave in a 2 nd order accurate fashion throughout the refinement, and the $L_{\infty}$ norm is appreciably reduced in the beginning stages of the refinement process.
These Figures indicate that the adaptive refinement would require approximately twice the number of cells than the structured solver for a given error magnitude. What is also indicated is that the adaptive mesh refinement requires approximately $2 / 3$ the number of cells for a given error norm than the uniformly refined (un-adapted) procedure.

TABLE III. Adaptively Refined Cartesian Error Norms

\begin{tabular}{|l|l|l|l|}
$\mathbf{N}$ & $L_{1}$ & $L_{2}$ & $L_{\infty}$ \\
\hline 118 & $5.345 \mathrm{e}-03$ & $8.658 \mathrm{e}-03$ & $4.497 \mathrm{e}-02$ \\
\hline 165 & $2.691 \mathrm{e}-03$ & $3.995 \mathrm{e}-03$ & $2.146 \mathrm{e}-02$ \\
\hline 337 & $1.310 \mathrm{e}-03$ & $1.693 \mathrm{e}-03$ & $6.658 \mathrm{e}-03$ \\
\hline 754 & $5.570 \mathrm{e}-04$ & $7.093 \mathrm{e}-04$ & $2.846 \mathrm{e}-03$ \\
\hline 1846 & $2.263 \mathrm{e}-04$ & $3.056 \mathrm{e}-04$ & $1.599 \mathrm{e}-03$ \\
\hline
\end{tabular}

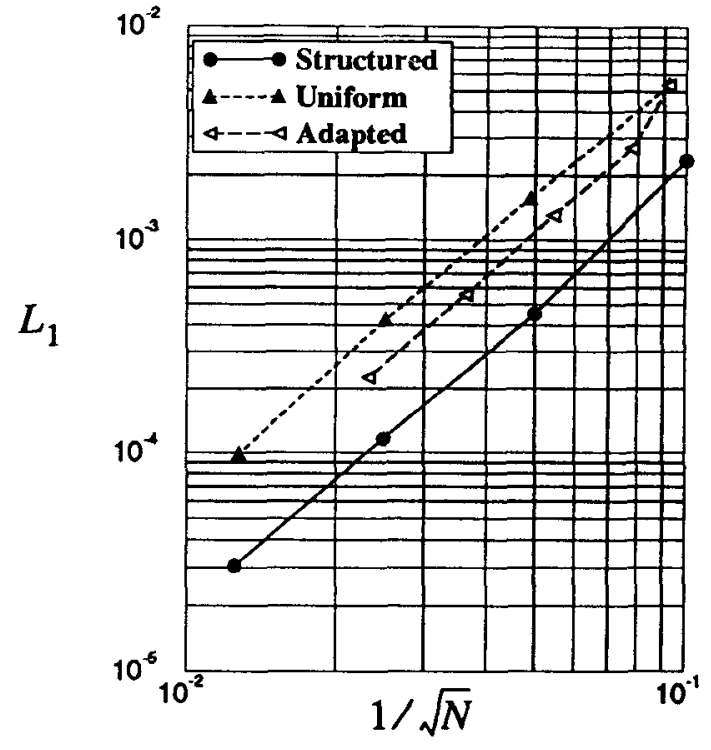

FIGURE 10. $L_{1}$ Norms of the error

To determine if the refinement strategy could be improved, the parameters that determine cell refinement and coarsening in equations 11 and 12 were adjusted. First, to see if the length scale weighting of the refinement criterion are not tuned properly, the length scale weight powers were changed to 1 and to 2 , but the effect was negligible. In addition, the cutoff parameters for coarsening and refining were adjusted. Cells were refined for refinement parameters greater than $1 / 2$ and $3 / 2$ times the standard deviation about zero, with no appreciable effect. Cells were coars- 
ened for refinement parameters less than $1 / 4$ and $1 / 2$ the standard deviation about zero (the default level is $1 / 10$ ), also to no effect. These results indicate that the refinement procedure is tuned properly for this smooth flow, and that no appreciable gains could be made with respect to the structured results.

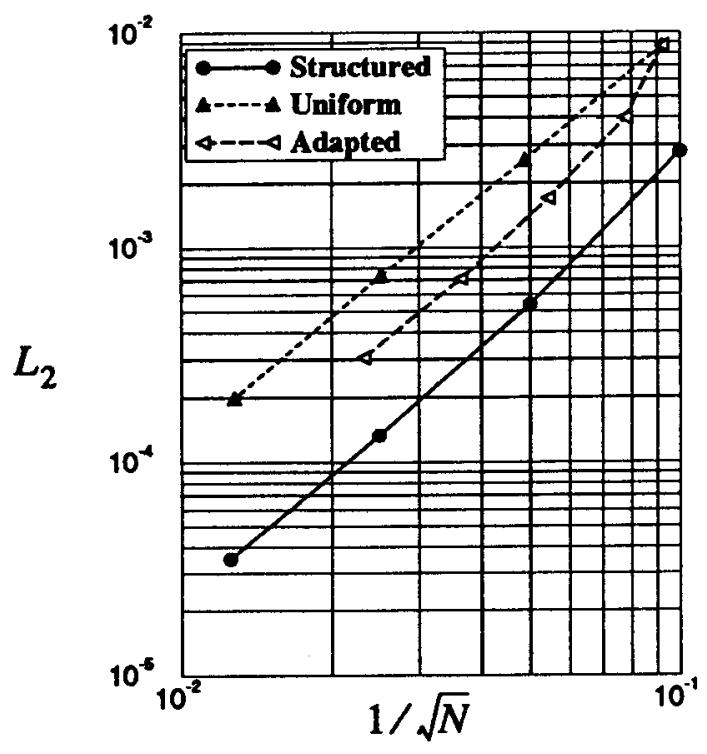

FIGURE 11. $L_{2}$ norm of the error

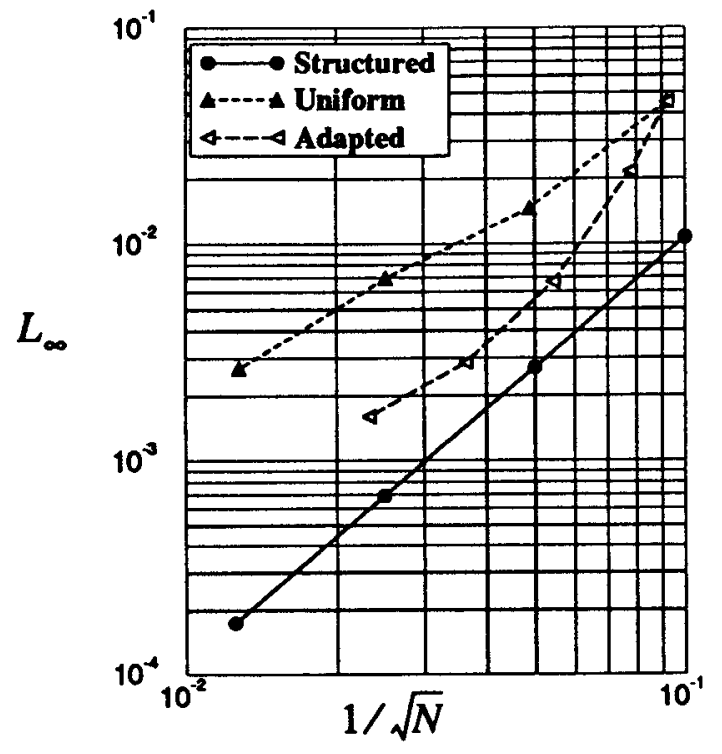

FIGURE 12. $L_{\infty}$ norm of the error
The fact that the Cartesian error is appreciably higher than the structured solver is disappointing, but if the particulars of the flow and the structured solver are taken into account, not too surprising. Ringleb's flow is a very smooth flow and has essentially a single length scale; it is surmised that on the structured grid, e ven a very coarse grid captures enough of the flow field so that refinement beyond this saturation will not yield much improvement over uniform refinement. In addition, the structured mesh used can be viewed as being in some sense "optimal"; not only is one family of the coordinate lines exactly aligned with the streamlines of the flow, but by virtue of the mesh generation, the mesh is denser in the region where the gradients are higher. Indeed, the grid alignment with the solution streamlines can be very beneficial since the Riemann solver used is only one-dimensional.

\section{III.d Green-Gauss/Minimum-Energy Reconstructions}

The Green-Gauss and Minimum-Energy reconstructions are evaluated for uniformly refined Cartesian-mesh solutions to Ringleb's flow. A truncation error analysis on uniform Cartesian grids indicates that the Green-Gauss has a slightly lower truncation error than the Minimum-Energy method, yet is still 2nd order accurate. This is borne out by the results shown in Figure 13, where all error norms are plotted versus the characteristic cell size for the GreenGauss and Minimum-Energy reconstructions.

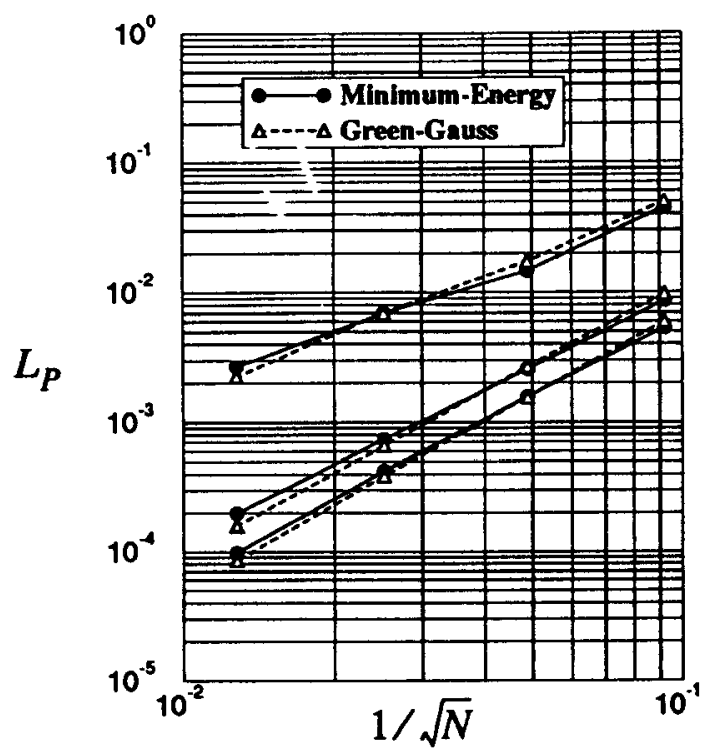

FIGURE 13. Comparison of Green-Gauss and Minimum-Energy Reconstructions 


\section{III.e Cut Cell Data Merging}

Cell merging is a process where small, cut cells on the mesh are merged into larger, neighboring cells. The Cartesian-mesh approach routinely generates meshes which have a many order variation of cell areas across the field. This wide area variation comes about through mesh refinement and from cell cutting. In the grid interior, smoothness can be imposed by only allowing a set number of tree level differences across faces. Smoothness can not be guaranteed at the boundaries. Typically, there are only a small number of very small cut cells introduced along the boundaries, where their areas may vary greatly compared to their neighbors. For steady state calculations, the stiffness introduced from these cells is reduced by using a spatially varying time step, but for time-accurate computations, as in [26] and [27], the efficiency can be greatly reduced. Cut cell merging is proposed to eliminate the stiffness caused by these few, very small cut cells that can be introduced in the mesh.

Cell merging creates a new cell, which we refer to as a merged cell, from a larger (mother) cell and a small cut (fragment) cell. This combination of the two mother and fragment cells into the single, larger merged cell can be achieved either geometrically or by a subtle change in the procedures used to compute the fluxes and flux balances on the fragment and mother cells. Since geometric merging can (except in fortunate circumstances) violate the hierarchy of the grid, we merge cells using the latter method. Theoretically, one can show that the global order of accuracy is unaffected by cell merging, although the error magnitude is increased. On a uniform mesh with an equal error distribution, one can rela.e the un-merged to merged $\mathrm{L}_{1}$ norm of the error as

$$
L_{1, \text { Merged }}=L_{1}\left(1+\frac{3 M}{N-M}\right)
$$

where $M$ cells have been merged on a mesh consisting of $\mathrm{N}$ cells prior to the cell merge. For small $\mathrm{M} / \mathrm{N}$, the magnitude of error is increased only negligibly, while the order is unaffected. The process of cell data merging proceeds as follows:

\section{Identification}

Fragment cells are chosen to be the minimum area cut cells in the mesh. Mother cells are chosen to be the maximum area cell that is a face neighbor to the fragment cell. For cells identified as merged cells, the merging changes its shape, and hence its centroid. So, the new merged cell centroid is computed from the fragment and mother cell centroids and areas.

$$
\bar{x}_{m}=\frac{\bar{x}_{M} A_{M}+\bar{x}_{F} A_{F}}{A_{M}+A_{F}}
$$

where the subscripts $m, M$ and $F$ refer to merged, Mother and Fragment cells, respectively.

\section{Reconstruction}

After all cell data has been reconstructed, the fragment cells data are altered so that at the interfaces between mother and fragment cells the reconstruction yields a unique value. That is, replace the fragment cell data as

$$
\begin{gathered}
\overline{\mathrm{u}}_{\mathrm{F}}=\overline{\mathrm{u}}_{M}+\nabla \mathrm{u}_{\mathrm{M}}\left(\overline{\mathrm{x}}_{\mathrm{F}}-\overline{\mathrm{x}}_{M}\right) \\
\nabla \mathrm{u}_{\mathrm{F}}=\nabla \mathrm{u}_{\mathrm{M}}
\end{gathered}
$$

\section{Flux Construction/Evolution}

The merged cell data is used as a matter of course in the flux computation for the mother and fragment cells. The flux balance on the larger, merged cell is obtained by summing the contributions of the mother and fragment cells to the merged cell

$$
R_{m}=R_{M}+\sum R_{F}
$$

where $R$ represents the flux balance of equation (10).

Here, we investigate the spatial accuracy of cell merging in the framework of Ringleb's flow. Adaptively refined calculations are made starting at a base grid level of $\mathrm{L}_{0}=4$ and refining 4 levels. At each refinement level, the smallest cut cell is merged into the largest of its neighbors. Figure 14 shows all error norms compared with the un-merged results presented in section III. In an attempt to gauge the increase in efficiency that could be achieved in a time accurate computation, the minimum time step is found for each grid level for the merged and un-merged results. The ratio of the minimum merged to minimum unmerged time step is shown in Figure 15, where 


$$
\lambda=\frac{\Delta t_{\text {min, merged }}}{\Delta t_{\text {min, un-merged }}}
$$

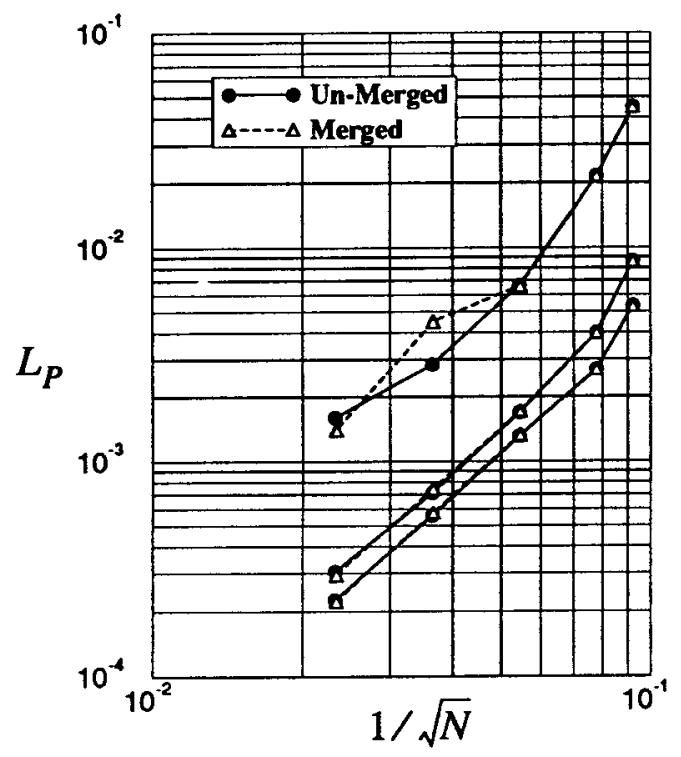

FIGURE 14. Error Norms: Merged and Un-Merged.

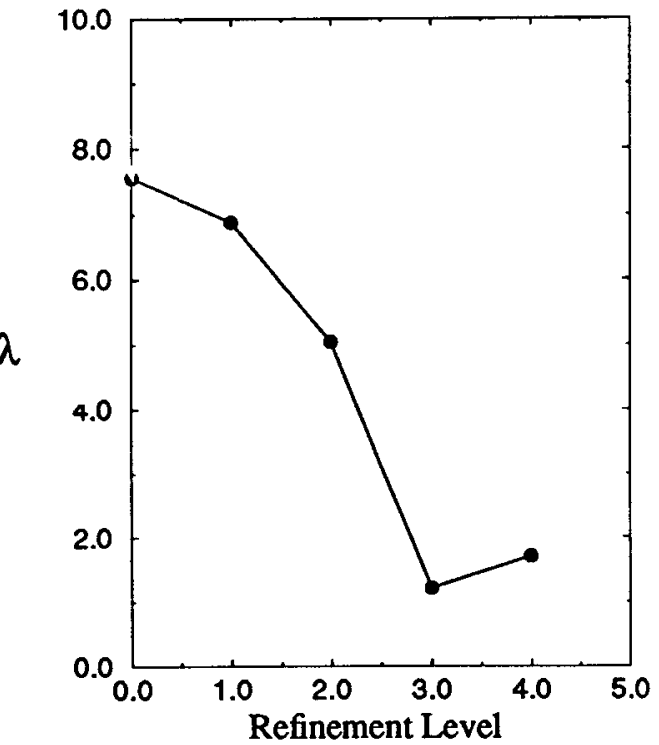

FIGURE 15. Ratio of Minimum Time Steps: Merged to Un-Merged.
One can see that the global error is practically unaffected by the cell merging, while there is a slight increase in the local error at the third refinement level. One can also see that the order of accuracy remains unchanged. The gain in efficiency through the increase of the time steps shown in Figure 15 drops off with refinement level. As the grid becomes populated with smaller and smaller cells from the adaptive mesh refinement, the smallest un-cut cell sizes approach the sizes of the small cut cells, causing the ratio of minimum step sizes to decrease.

\section{Accuracy Assessment: Non-Smooth Flow}

One of the great promises of adaptive mesh refinement is to achieve a high level of resolution and accuracy using a minimum of resources. But, as is shown in the preceding example, for a flow with a single length scale, or one that is fairly uniform, it is hard to beat uniform mesh refinement. The following example shows that for the proper type of flow field, adaptive mesh refinement can give an appreciable gain in performance over uniform mesh refinement. For this study, the supersonic flow through a stylized axi-symmetric inlet is computed using the Cartesian-cell approach on a sequence of uniformly and adaptively refined meshes. The inlet studied is based upon the mixed compression inlet investigated in [25]. This inlet is designed to decelerate a $M_{\infty}=2.5$ flow through a series of oblique shock waves which terminate at a normal shock wave in the diffuser. For the study here, the free stream Mach number is reduced to $M_{\infty}=2.0$ and the cowl is displaced radially outward $1 / 2$ cowl radius from the centerline. Extrapolation type procedures are applied at the exit boundaries, yielding a supersonic flow throughout the inlet. The centerbody and inner cowl surface shapes are made using the curve fits supplied in [25], while the outer cowl surface is stylized for this study. Uniformly refined computations were made for base grid levels $\mathrm{L}_{0}=6$ through $\mathrm{L}_{0}=9$. Adaptively refined calculations were made starting at a grid base level $L_{0}=6$ and refining 5 levels. Figures 16 and 17 show computed pressure contours and the resulting adapted grid corresponding to 3 refinement levels below the base mesh. We assess grid convergence by comparing the drag coefficients of the uniformly and adaptively refined calculations, shown in Figure 18. 


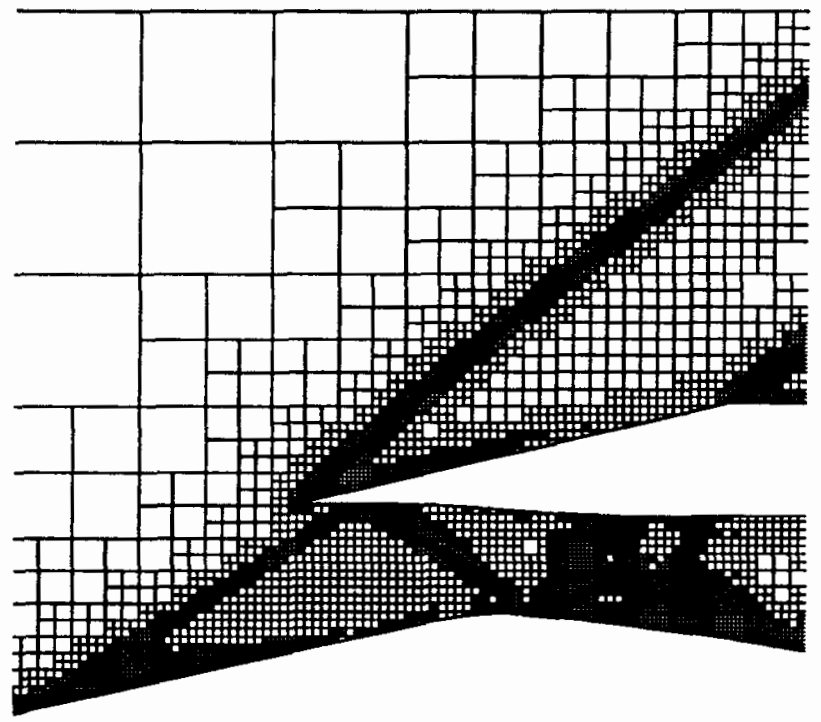

FIGURE 16. Adapted Grid: Axi-Symmetric Inlet

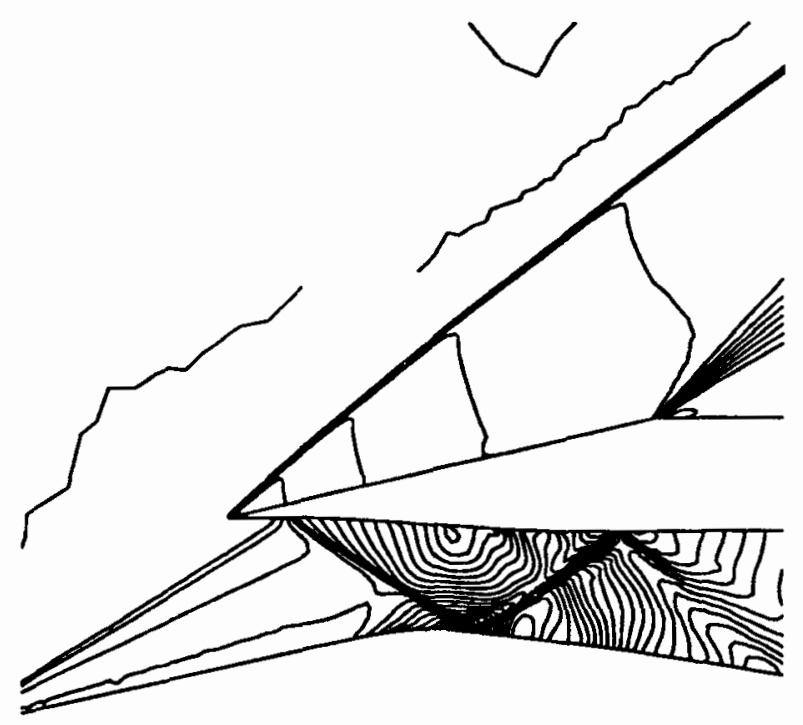

FIGURE 17. Adapted Grid: Pressure Contours

As can be seen from the figure, the adaptively refined calculations become grid converged within approximately 25,000 cells. The uniformly refined drag coefficients appear to be trying to reach the same asymptote, although for this fine, uniform grid of over 150,000 cells, does not appear to have reached grid convergence.

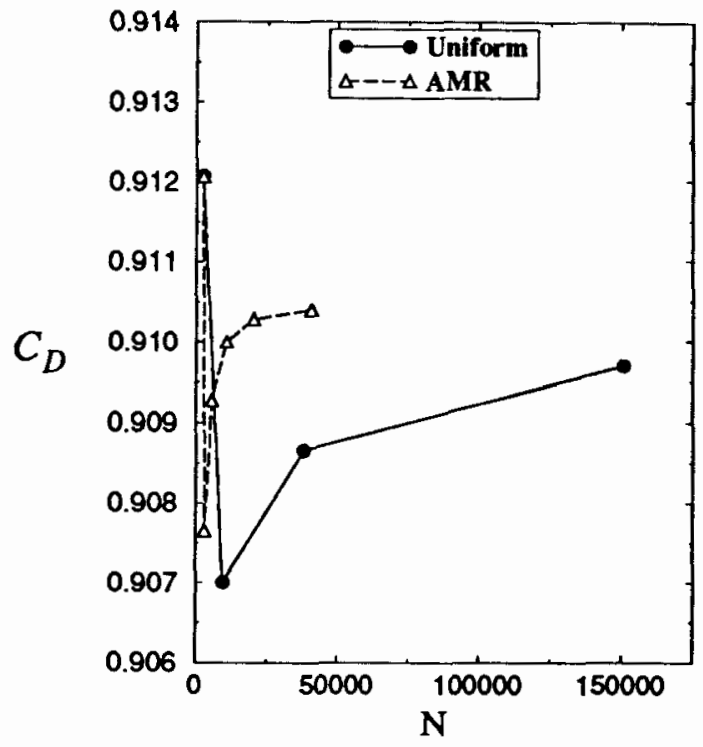

FIGURE 18. Adaptively and Uniformly Refined Drag Coefficients

\section{Conclusions}

In this paper a critical assessment of the accuracy of Cartesian-mesh approaches has been made. We have explained the basics behind the hierarchical data structure that can be used to store the mesh data and how arbitrary geometries can be represented with the Cartesian-mesh approach. The Cartesian-mesh approach presented here used a cell centered, upwind based finite-volume formulation coupled with a particular $\mathrm{K}$-exact reconstruction of the primitive variables. Adaptive mesh refinement was achieved using cell size scaled refinement parameters based upon the local compressibility and rotationality of the fluid.

An exact solution to the Euler equations (Ringleb's flow) was used to assess the order and magnitude of the solution error. The solution errors for both structured and Cartesian-mesh based calculations of Ringleb's flow were compared directly. Uniform refinement was performed for the structured-mesh calculations while both uniform and solution adaptive refinements were made for the Cartesianmesh approach.

The assessment indicated that the Cartesian-mesh approach used here was globally second-order accurate, and remained so through adaptive refinement, while the local error was better than first order accurate, but less than second order. Adaptive mesh refinement was criti- 
cally evaluated for Ringleb's flow, which is smooth and analytic, and for a very non-smooth flow; the supersonic flow through a mixed-compression, axi-symmetric inlet. For the smooth flow, the adaptive mesh refinement was shown to not give a gain in accuracy for given resources over the uniformly refined calculations. For the nonsmooth flow, the adaptive mesh refinement was shown to give a large gain in efficiency over the uniformly refined procedure. These results indicate that adaptive mesh refinement is best suited for non-smooth flows and/or flows with widely varying length scales.

The accuracy of two different reconstruction procedures, Minimum-Energy and Green-Gauss, were compared directly and shown to give no appreciable difference. Cell data merging was investigated and was shown to increase the efficiency for time accurate computations by increasing the allowable time step with a negligible cost in accuracy.

\section{Acknowledgments}

The authors would like to express their gratitude to Professor M.J. Berger for her insightful comments regarding this work. Thanks also go to Mr. B.P. Curlett for use of his workstation "homet" for some of the Ringleb flow calculations, and to Dr. D. Rigby, Dr. P. Jorgenson, Dr. J. Lee and Mr. C. Steffen, Jr. for many interesting discussions.

\section{Bibliography}

[1] Dannenhoffer, J.F., III, “A Comparison of Adaptivegrid Redistribution and Embedding For Steady Transonic Flows", International Journal for Numerical Methods in Engineering, Vol. 32, 1991, pp. 653-663.

[2] Berger, M.J., “Adaptive Mesh Refinement for Hyperbolic Partial Differential Equations”, Joumal of Computational Physics, Vol. 53, 1984, pp. 484512.

[3] Berger, M.J. and Jameson, A., “Automatic Adaptive Grid Refinement for the Euler Equations", AIAA Journal, Vol. 23, No. 4, 1985, pp. 561-568.

[4] Berger, MJ. and Colella, P., "Local Adaptive Mesh Refinement for Shock Hydrodynamics", Journal of Computational ysics, Vol. 82, 1989, pp. 64-84.
[5] Quirk, J.J., "An Adaptive Grid Algorithm for Computational Shock Hydrodynamics", Ph.D. Thesis, Cranfield Institute of Technology, College of Aeronautics, 1991.

[6] Young, D.P, Melvin, R.G., Bieterman, M.B., Johnson, F.T., Samant, S.S. and Bussoletti, J.E., "A Locally Refined Rectangular Grid Finite Element Method: Application to Computational Fluid Dynamics and Computational Physics", Journal of Computational Physics, Vol. 92, 1991, pp. 1-66.

[7] Clarke, D.K., Salas, M.D., and Hassan, H.A., "Euler Calculations for Multielement Airfoils Using Cartesian Grids", AIAA Journal, Vol. 24, No. 3, 1986, pp 353358.

[8] Quirk, J.J., “An Alternative to Unstructured Grids for Computing Gas Dynamic Flows Around Arbitrarily Complex Two-Dimensional Bodies", ICASE Report No. 92-7.

[9] Berger, M.J., LeVeque, R.J., “An Adaptive Cartesian Mesh Algorithm for the Euler Equations in Arbitrary Geometries", AIAA Paper 89-1930-CP.

[10]Berger, M.J., LeVeque, R.J., "Stable Boundary Conditions for Cartesian Grid Calculations", Computing Systems in Engineering, Vol. 1, Nos. 2-4, pp. 305-311, 1990.

[11]Berger, MJ., LeVeque, R.J., "A Rotated Difference Scheme for Cartesian Grids in Complex Geometries", AIAA Paper CP-91-1602.

[12]Morinishi, K., "A Finite Difference Solution of the Euler Equations on Non-Body Fitted Cartesian Grids", Computers Fluids, Vol. 29, No. 3, pp. 331-344, 1992.

[13]Epstein, B., Luntz, A.L., Nachson, A., "Multigrid Euler Solver About Arbitrary Aircraft Configurations with Cartesian grids and Local Refinement", AIAA Paper 89-1960-CP.

[14]De Zeeuw, D., and Powell, K.G., "An Adaptively Refined Cartesian Mesh Solver for the Euler Equations", To appear in Journal of Computational Physics, 1991.

[15]De Zeeuw, D., and Powell, K.G., "Euler Calculations of Axisymmetric Under-Expanded Jets by an Adaptive-Refinement Method", AIAA Paper 93-0321.

[16]Barth, T.J., "On Unstructured Grids and Solvers", Computational Fluid Dynamics Lecture Series, 199003, Von Karman Institute for Fluid Dynamics. 
[17]Barth, T.J., and Frederickson, P.O., "Higher Order Solution of the Euler Equations on Unstructured Grids Using Quadratic Reconstruction", AIAA Paper 90-0013.

[18]Powell, K.G., Roe, P.L. and Quirk, J.J., “AdaptiveMesh Algorithms for Computational Fluid Dynamics", Presented at the workshop Algorithmic Trends in Computational Fluid Dynamics in the 1990's, Sept. 1991. (To appear in published proceedings, Springer-Verlag).

[19]Godfrey, A.G., Mitchell, C.R., Walters, R.W. "Practical Aspects of Spatially High Accurate Methods", AIAA Paper 92-0054.

[20]Roe, P.L., “Approximate Riemann Solvers, Parameter Vectors and Difference Schemes", Journal of Computational Physics, Vol. 43, pp 357-372, 1981.

[21]Paillere, P.H., Powell, K.G. and De Zeeuw, D., "A Wave-model Based Refinement Criterion for Adaptive-Grid Computation of Compressible Flows", AIAA Paper 92-0322.

[22]"Test Cases for Inviscid Flow Field Methods: Report of Fluid Dynamics Panel Working Group 07", AGARD Report No. AR-211.

[23]Halt, D.W. and Agarwal, R.K., "Compact Higher Order Characteristic-Based Euler Solver for Unstructured Grids", AIAA Journal, Vol. 30, No. 8, Aug. 1992, pp. 1993-1999.

[24]Chima, R.V., "Viscous Three-Dimensional Calculations of Transonic Fan Performance", NASA TM 103800.

[25]Hingst, W.R., and Johnson, D.F, "Experimental Investigation of Boundary Layers in an Axisymmetric, Mach 2.5, Mixed-Compression Inlet", NASA TM X-2902.

[26]Bayyuk, S.A., Powell, K.G. and van Leer, B., "An Algorithm For the Simulation of 2-D Unsteady Inviscid Flows Around Arbitrarily Moving and Deforming Bodies of Arbitrary Geometry", in AIAA 11th Computational Fluid Dynamics Proceedings.

[27]Chiang, Yu-Liang, "Simulation of Unsteady Inviscid Flow On An Adaptively Refined Cartesian Grid", Ph.D. Thesis, University of Michigan, Aerospace Engineering Department, 1992. 